\title{
LIVER FUNCTION ABNORMALITIES IN HIV POSITIVE INDIVIDUALS WITH VIRAL HEPATITIS CO- INFECTION
}

\author{
R. B. Sudagar Singh ${ }^{1}$, K. Vasanthan'2, Vengadakrishnan K3, J. Damodharan ${ }^{4}$ \\ ${ }_{1}^{1}$ Associate Professor, Department of General Medicine, Sri Ramachandra Medical College and Research Institute, Chennai. \\ ${ }^{2}$ Associate Professor, Department of General Medicine, Sri Ramachandra Medical College and Research Institute, Chennai. \\ ${ }^{3}$ Professor, Department of General Medicine, Sri Ramachandra Medical College and Research Institute, Chennai. \\ ${ }^{4}$ Professor, Department of General Medicine, Sri Ramachandra Medical College and Research Institute, Chennai.
}

\begin{abstract}
BACKGROUND: Liver enzyme elevations are common in human immunodeficiency virus (HIV)-infected patients, and their diagnosis and management becomes a challenge because of complex pathogenic mechanisms. Liver enzyme abnormalities may be worsened by hepatitis B (HBV) or hepatitis C (HCV) infection, which each have their own risks for chronic immune-mediated liver disease along with direct cytotoxicity.

OBJECTIVES: The present study was done to identify various liver function abnormalities in HIV and viral hepatitis co- infection METHODS: The study included 22 HIV seropositive patients along with serology positive for hepatitis (19 patients were HBsAg positive and 3 patients were HCV positive) who presented to Sri Ramachandra Medical College and Hospital from the year 2012 to 2014. Complete haemogram, blood urea, serum creatinine, liver function tests, CD4 count, and ultrasound abdomen was done for all the patients. LFT abnormalities were compared with HIV positive patients without hepatitis (78 patients).

RESULTS: LFT abnormalities were significant in comparison to patients with negative hepatitis serology. Only a modest elevation of liver enzymes was seen in hepatitis. Co-infection. Majority of the patients had elevation of transaminases and INR was normal in all HCV positive patients.
\end{abstract}

CONCLUSION: HBV and HCV coinfection increases the incidence of LFT abnormality. Only a modest elevation of liver enzymes was seen in hepatitis co-infection.

KEYWORDS: HIV Infection, Viral Hepatitis, Liver Function.

HOW TO CITE THIS ARTICLE: R. B. Sudagar Singh, K. Vasanthan, Vengadakrishnan. K, J. Damodharan. "Liver Function Abnormalities in HIV Positive Individuals with Viral Hepatitis Co-Infection". Journal of Evolution of Medical and Dental Sciences 2015; Vol. 4, Issue 91, November 12; Page: 15691-15693, DOI: 10.14260/jemds/2015/2258.

INTRODUCTION: Liver enzyme elevations are common in human immunodeficiency virus (HIV)-infected patients, and their diagnosis and management becomes a challenge because of complex pathogenic mechanisms. Hepatotoxicity related to the highly active antiretroviral therapy (HAART) regimen, idiosyncratic or immunoallergic mechanisms, and direct cytotoxicity enhanced by an underlying liver disease all contribute to liver injury. Liver enzyme abnormalities may be worsened by hepatitis B (HBV) or hepatitis C (HCV) infection, which each have their own risks for chronic immune-mediated liver disease along with direct cytotoxicity.[1] At the beginning of the AIDS era, liver dysfunction in HIV -infected patients mainly corresponded with opportunistic infections (e.g., with cytomegalovirus [CMV], mycobacteria, leishmaniasis, cryptosporidiosis and microsporidiosis), tumors (Lymphoma and Kaposi sarcoma); and drug-related hepatitis (Caused by trimetoprimsulfamethoxazole and other antibiotics).

Financial or Other, Competing Interest: None.

Submission 17-10-2015, Peer Review 18-10-2015,

Acceptance 29-10-2015, Published 11-11-2015.

Corresponding Author:

Dr. Vengadakrishana. $K$,

Department of General Medicine,

Sri Ramachandra Medical College and Research Institute,

Chennai.

E-mail:drkvk1975@gmail.com

DOI:10.14260/jemds/2015/2258.
HAART has changed the pattern of hepatic events in HIV infection, and the liver is an important organ to consider when treating HIV-infected patients. Human immunodeficiency virus (HIV) infection of liver cells can contribute to liver disease progression by either direct or indirect mechanisms. HIV can directly infect hepatocytes, hepatic stellate cells (HSCs) and Kupffer cells (KCs). Glycoprotein120 binding to CXCR4 can induce hepatocyte apoptosis and activation of HSCs which in turn induce fibrosis. Nucleoside reverse transcriptase inhibitors (NRTIs) and HIV itself [Via peroxisome proliferatoractivated receptor (PPAR) effects] may also contribute to liver disease by inducing the metabolic syndrome.

HIV infection of the gastrointestinal tract leads to an increase in lipopolysaccharide (LPS) which can stimulate hepatocytes, KCs and HSCs to produce pro-inflammatory cytokines and chemokines which attract activated lymphocytes and monocytes to the liver which may further drive fibrosis. HIV infection of the gastrointestinal tract leads to an increase in lipopolysaccharide (LPS) which can stimulate hepatocytes, KCs and HSCs to produce pro-inflammatory cytokines and chemokines which attract activated lymphocytes and monocytes to the liver which may further drive fibrosis.[2,3,4,5,6] Hepatocyte apoptosis can trigger profibrotic activity of HSC, as has been demonstrated in both HIVHBV co-infection and HIV-HCV co-infection.[7]

The exact role of HIV-induced hepatic cell apoptosis and liver disease needs to be determined by further studies. The present study was done to identify various liver function abnormalities in HIV and viral hepatitis co infection. 
MATERIAL AND METHODS: The study included 22 HIV positive patients along with serology positive for hepatitis (19 patients were HBsAg positive and 3 patients were $\mathrm{HCV}$ positive) presented to Sri Ramachandra Medical College and Hospital from the year 2012 to 2014, after approval of Hospital ethics committee. All patients fulfilling inclusion criteria (All HIV seropositive patients with serology positive for hepatitis $\mathrm{B}$ or C confirmed by ELISA) were screened and investigations done. Patients with age less than 18 years and pregnant patients were excluded from the study.

A questionnaire for detailed history was taken from all patients and physical examination was done. A thorough abdomen examination to demonstrate hepatomegaly, splenomegaly, free fluid, cirrhosis of liver was carried out. Complete blood count, blood urea, sugar, serum creatinine, liver function tests including coagulation profile and urine analysis were done for all patients. The CD4 lymphocyte count was done by flow cytometry method. Abnormal liver function tests were defined as $>1.25$ ULN in HIV positive patients. The data were compared with HIV positive patients without hepatitis (78 patients).

The collected data was analyzed using Statistical Package of Social Sciences (SPSS) 17.0 for windows. Data were expressed as the mean + standard deviation. A p value of less than 0.05 was considered statistically significant.

RESULTS: The study included 19 patients with HBsAg positive and 3 patients with HCV positive. 78 patients had serology negative for hepatitis B or C. Various liver parameters were analysed in patients with hepatitis co-infection and data compared with patients having no hepatitis. The results are summarised in tables 1 and 2. Only SGOT elevation in hepatitis B co-infection had statistical significance.

DISCUSSION: The study was done to ascertain whether LFT abnormalities would be significantly affected with hepatitis co-infection. Direct injury by HIV can cause abnormal liver function. Co-infection with viral hepatitis could further worsen the liver function. In the present study 19 patients (19\%) were positive for HBV surface antigen and 3 patients $(3 \%)$ had antibodies to HCV.

In the study by Richard K. Sterling et al..[8] 7.1\% patients were positive for HBV surface antigen and $24 \%$ patients had antibodies to HCV. In the study by Ejilemele AA et al..[9] 2.3\% were $\mathrm{HBsAg}$ positive and $0.8 \%$ patients had antibodies to HCV. In the study done by Ponsiano Ocama et al.[10] 15\% patients were HBsAg positive and 3\% patients had antibodies to HCV. In our study the prevalence of those with HBV and HCV was SGOT-89.5\%, SGPT-73.7\%, ALP-52.6\% compared to SGOT$61.7 \%$, SGPT-56.8\%, ALP-32.1\% in those without HBV and HCV co-infection.

In the study done by Richard K. Sterling et al. the prevalence of those with HBV and HCV was SGOT - 64\%, SGPT46\%, ALP-63\% compared to SGOT-20\%, SGPT-15\%, ALP- 43\% in those without HBV and HCV co-infection.
In our study there was a statistically significant relationship between Hepatitis B positive status and SGOT elevation (p-value- 0.021).

CONCLUSION: LFT abnormalities were significant in comparison to patients with negative hepatitis serology. Only a modest elevation of liver enzymes was seen in hepatitis coinfection. HBV and HCV co-infection increases the incidence of LFT abnormality.

\section{BIBLIOGRAPHY:}

1. Reciprocal Interactions between Human immunodeficiency virus and hepatitis $\mathrm{C}$ virus infections. Clin Infect Dis 1996;23:1117-25.

2. Soriano $\mathrm{V}$ et al. Impact of chronic viral liver disease due to hepatitis viruses as cause of hospital admission and death in HIV-infected drug users: Eur J Epidemiol 1999;15:1-4.

3. Macias J, Japón MA, Sáez C, Palacios RB et al. Increased hepatocyte fas expression and apoptosis in HIV and hepatitis C virus coinfection: J Infect Dis. 2005;192:1566 $-1576$.

4. Tuyama AC, Hong F, Saiman Y, Wang C, Ozkok D, Mosoian A, Chen P, Chen BK, Klotman ME, Bansal MB. Human immunodeficiency virus (HIV)-1 infects human hepatic stellate cells and promotes collagen I and monocyte chemoattractant protein -1 expression: implications for the pathogenesis of HIV/hepatitis C virus-induced liver fibrosis: Hepatology, 2010;52:612 - 622 .

5. Balagopal A, Philp FH, Astemborski J et al. Human immunodeficiency virus-related microbial translocation and progression of hepatitis C. Gastroenterology: 2008;135:226 -233.

6. Tan AT, Koh S, Goh W, Zhe HY et al. A longitudinal analysis of innate and adaptive immune profile during hepatic flares in chronic hepatitis B: J Hepatol. 2010;52:330 -339.

7. Iser DM, Avihingsanon A, Wisedopas $\mathrm{N}$ et al. Increased intrahepatic apoptosis but reduced immune activation in HIV-HBV co-infected patients with advanced immunosuppression: AIDS. 2011;25:197-205.

8. RK Sterling, S Chiu, K Snider, D Nixon, The prevalence and risk factors for abnormal liver enzymes in HIVpositive patients without hepatitis B or C coinfections: Digestive diseases and sciences, 2008-Springer.

9. AA Ejilemele, CA Nwauche, OA Ejele. Pattern of abnormal liver enzymes in HIV patients presenting at a Nigerian Tertiary Hospital: The Nigerian postgraduate journal, 2007- europepmc.org.

10. Ocama.P, Katwere.M, Piloya.T et al. The spectrum of liver diseases in HIV infected individuals at an HIV treatment clinic in Kampala, Uganda: Afr Health Sci. 2008;8:8 -12. 


\begin{tabular}{|c|c|c|c|}
\hline Parameter & Hepatitis B Negative & Hepatitis B Positive & P value \\
\hline Bilirubin $<1.5$ & 72 & 16 & 0.572 \\
\hline Bilirubin $>1.5$ & 9 & 3 & 0.572 \\
\hline SGOT $<44$ & 31 & 2 & 0.021 \\
\hline SGOT $>44$ & 50 & 17 & 0.021 \\
\hline SGPT $<51$ & 35 & 5 & 0.176 \\
\hline SGPT $>51$ & 46 & 14 & 0.176 \\
\hline Alkaline phosphatase $<161$ & 55 & 9 & 0.803 \\
\hline Alkaline phosphatase $>161$ & 26 & 10 & 0.803 \\
\hline INR $<1.1$ & 53 & 12 & 0.952 \\
\hline INR $>1.1$ & 28 & 7 & 0.952 \\
\hline Albumin $>3.2$ & 51 & 10 & 0.406 \\
\hline Albumin $<3.2$ & 30 & 9 & 0.406 \\
\hline \multicolumn{2}{|l|}{ Table 1: HIV and Hepatitis B co-infection } \\
\hline
\end{tabular}

LFT elevations were significant in patients with hepatitis B co-infection. Statistical significance was seen only for SGOT.

\begin{tabular}{|c|c|c|c|}
\hline Parameter & Hepatitis C Negative & Hepatitis C Positive & P value \\
\hline Bilirubin $<1.5$ & 85 & 3 & 0.566 \\
\hline Bilirubin $>1.5$ & 12 & 0 & 0.566 \\
\hline SGOT $<44$ & 32 & 1 & 0.990 \\
\hline SGOT $>44$ & 65 & 2 & 0.990 \\
\hline SGPT $<51$ & 38 & 2 & 0.338 \\
\hline SGPT $>51$ & 59 & 1 & 0.338 \\
\hline Alkaline phosphatase $<161$ & 62 & 2 & 0.992 \\
\hline Alkaline phosphatase $>161$ & 35 & 1 & 0.992 \\
\hline INR $<1.1$ & 62 & 3 & 0.197 \\
\hline INR $>1.1$ & 35 & 0 & 0.197 \\
\hline Albumin $>3.2$ & 60 & 1 & 0.318 \\
\hline Albumin $<3.2$ & 37 & 2 & 0.318 \\
\hline \multicolumn{2}{|c|}{ Table 2: HIV and Hepatitis C co-infection } \\
\hline
\end{tabular}

The analysed data showed that LFT abnormalities were not statistically significant in HCV co-infection. 